\title{
Anharmonic surface vibrations in photoemission from alkali metals
}

\author{
G. K. Wertheim, D. M. Riffe, ${ }^{*}$ and P. H. Citrin \\ AT\&T Bell Laboratories, Murray Hill, New Jersey 07974 \\ (Received 27 September 1993)
}

\begin{abstract}
The phonon widths of outermost core-electron photoemission spectra from (110)-oriented overlayers of $\mathrm{Na}, \mathrm{K}$, and $\mathrm{Rb}$ metals show the expected Debye behavior for the bulk atoms, but significant deviations for the surface atoms. The data indicate a softening of the surface vibrational mode above $200 \mathrm{~K}$. This effect, which is weak in $\mathrm{Na}$ but strong in $\mathrm{K}$ and $\mathrm{Rb}$, demonstrates that the vibrational mode normal to the surface is anharmonic.
\end{abstract}

\section{INTRODUCTION}

It has long been recognized that the vibrations of atoms in the surface of a bulk solid are anisotropic, ${ }^{1-4}$ with a larger amplitude normal to the surface. This generalization is not valid when foreign atoms are adsorbed on the surface. More recently, surface anharmonicity has been invoked to explain many different observations, including increased thermal expansion of the surface layer, ${ }^{5}$ loss of intensity of surface (inverse) photoemission peaks, ${ }^{6-8}$ and anisotropic Debye-Waller damping in surface extended $x$-ray-absorption fine structure from adsorbates $^{9-11}$ and adsorbate-covered metal surface atoms. ${ }^{12}$ Thermal effects on the intensity of He-atom scattering peaks from the $\mathrm{Cu}(110)$ surface have been variously attributed to thermal roughening ${ }^{13}$ and surface anharmonicity. ${ }^{14}$ The latter effect has recently been observed in electron-energy-loss data from that surface. ${ }^{15}$

Here, we present the results of a photoemission study of alkali metals in which the effects of anharmonicity on phonon widths of the surface-atom peaks clearly emerge. Following up on our demonstration of a soft phonon mode normal to the surface of $\mathrm{Na},{ }^{16}$ we have extended out study to $\mathrm{K}$ and $\mathbf{R b}$ over the same temperature range. The main difference between $\mathrm{Na}$ and these two heavier alkalis lies in their smaller cohesive energy and larger vapor pressure. We find, for all three metals, anharmonicity of the vibrational mode associated with surface atoms moving normal to the (110) surface. This anharmonicity, which becomes apparent in our measurements at temperatures greater than half of the melting point, is the result of anisotropic mode softening implicit in the desorption channel for surface atoms.

\section{EXPERIMENT}

The alkali-metal samples were prepared by condensation of metal vapor, obtained from commercial SAES Getters sources, onto a $\mathrm{Ni}(100)$ substrate cooled to 78 K. ${ }^{16}$ Low-energy electron diffraction (LEED) from the $\mathrm{Na}$ sample showed a single-domain (110) orientation. $^{15}$ LEED from the $\mathrm{K}$ films showed no long-range order. The lack of observable LEED features for $K$ in this temperature range is attributed to thermal broadening, since a $K$ film at $20 \mathrm{~K}$, prepared as in this work, exhibits a well- defined (110) diffraction pattern. ${ }^{17}$ LEED observations of the $\mathbf{R b}$ sample were not made. However, the core-level data, which exhibit only two peaks, are indicative of (110) orientation for this surface as well.

Photoemission data were obtained on the AT\&T Bell Laboratories-University of Oregon $6 \mathrm{~m}$ toroidal grating monochromator beam line at the National Synchrotron Light Source. The photoelectron energy distributions were measured with a $100-\mathrm{mm}$ Vacuum Science Workshop hemispherical analyzer operated with a pass energy of $2 \mathrm{eV}$, yielding nominal resolutions of $40 \mathrm{meV}$. The monochromator resolution was in the range from 50 to $80 \mathrm{meV}$, depending on photon energy, giving total instrumental resolutions in the range of $65-90 \mathrm{meV}$. Ambient pressure was in the range of $(1-2) \times 10^{-10}$ Torr, dominated by $\mathrm{H}_{2}$. The temperature-dependent data were taken while the substrate warmed up over a period of $2 \mathrm{~h}$, up to the point where desorption becomes appreciable, i.e., up to a vapor pressure of $3 \times 10^{-11}$ Torr.

\section{RESULTS AND DISCUSSION}

The data were analyzed by fitting them with spin-orbit (SO) doublets made up of Doniach-Šunjić ${ }^{18}$ lines. The background was represented by a sloping line and a power-law term. We initially assumed that the bulk and surface SO splittings are the same, and that the natural lifetime widths of the $p_{3 / 2}$ and $p_{1 / 2}$ core holes at the surface are the same as in the bulk. Only in the case of $R \mathbf{b}$, where the four SO lines are resolved at low temperature, was it necessary to modify one of these assumptions, namely that regarding the SO splitting. The data convincingly show that the surface SO splitting is $10 \mathrm{meV}$ smaller than that of the bulk. For $\mathrm{Na}$ and $\mathrm{K}$, in which the bulk $p_{1 / 2}$ and surface $p_{3 / 2}$ lines overlap, the bulk SO ratio was constrained to the statistical value of 0.5 because it could not be determined from the data. The singularity indices and Gaussian widths of the bulk and surface components were assigned independent values from previous work. ${ }^{19}$

In order to determine the best value for such parameters as the natural widths, singularity indices, surfaceatom core-level shift, and SO splitting, all of which are clearly temperature independent, ${ }^{20}$ we first fit the entire set of temperature-dependent data for each metal without 
constraints beyond those already mentioned. By progressively constraining those parameters which are independent of temperature, we arrive at a set of values which yields satisfactory fits to all the data when only the bulk and surface Gaussian widths remain free. The parameters used in the final analysis are summarized in Table I. This iterative procedure reduces the errors in the Gaussian width due to the coupling with other parameters, which becomes increasingly troublesome at the higher temperatures.

Analysis of a representative $\mathrm{K} 3 p$ spectrum is illustrated in Fig. 1. The data, taken at $172 \mathrm{~K}$, were fitted using the parameters in Table $I$, and are one of a series of data sets taken as a function of temperature. The SO splitting obtained in this analysis is found to be identical to the value for the neutral $\mathrm{K}$ atom with $3 p^{5} 4 s^{2}$ configuration, ${ }^{21}$ indicating that the $3 p$ hole in the metal is fully screened. The value of the surface-atom core-level shift has been confirmed by SO stripping data taken at higher photon energy. The larger $p_{1 / 2}$ lifetime width relative to that for the $p_{3 / 2}$ state is due to Coster-Kronig transitions, while the larger singularity index for the surface atoms is due to increased contribution of $s$-wave scattering in the narrower, more atomiclike surface conduction band. ${ }^{19}$ The only unusual aspect of the $\mathrm{K}$ data is the nonstatistical SO ratio for the surface atoms. This ratio has been found to depend on the photoelectron take-off angle, a phenomenon not unique to this metal and one that is presently not understood.

Representative results for the $\mathrm{Rb} 4 p$ data taken at 147 $K$ are illustrated in Fig. 2. The parameters for $R b$ in Table $I$ are in good agreement with those recently obtained from data taken with $\mathrm{He}$ II resonance radiation. ${ }^{22}$ The Na data of Ref. 16, including additional data obtained at higher temperatures, were reanalyzed using the method described here, yielding results very similar to those reported in that earlier work.

The total Gaussian widths for the bulk and surface components in the three metals are plotted in Fig. 3 as the square of the width versus temperature. This presentation is motivated by the high-temperature expansion of the expression for the phonon width in the Debye model. ${ }^{16}$ For temperatures above the Debye temperature, $\Theta_{D}$, the total measured Gaussian width $G_{\text {tot }}$, including

TABLE I. Binding energies, surface-atom core-level shifts, and line-shape parameters used in the analysis of the $\mathrm{Na}, \mathrm{K}$, and Rb spectra.

\begin{tabular}{lccc}
\hline \hline & $\mathrm{Na}$ & $\mathrm{K}$ & $\mathrm{Rb}$ \\
\hline Binding energy (eV) & 30.6 & 18.34 & 14.93 \\
Surface shift (meV) & 184 & 175 & 182 \\
Natural width $p_{3 / 2}(\mathrm{meV})$ & 12 & 14 & 20 \\
Natural width $p_{1 / 2}(\mathrm{meV})$ & 12 & 50 & 95 \\
Singularity index (bulk) & 0.16 & 0.14 & 0.14 \\
Singularity index (surface) & 0.20 & 0.21 & 0.22 \\
SO splitting (bulk) (meV) & 160 & 257 & 856 \\
SO splitting (surface) (meV) & 160 & 257 & 846 \\
SO ratio (bulk) & 0.50 & 0.50 & $\sim 0.52$ \\
SO ratio (surface) & 0.56 & 0.60 & $\sim 0.55$ \\
\hline \hline
\end{tabular}

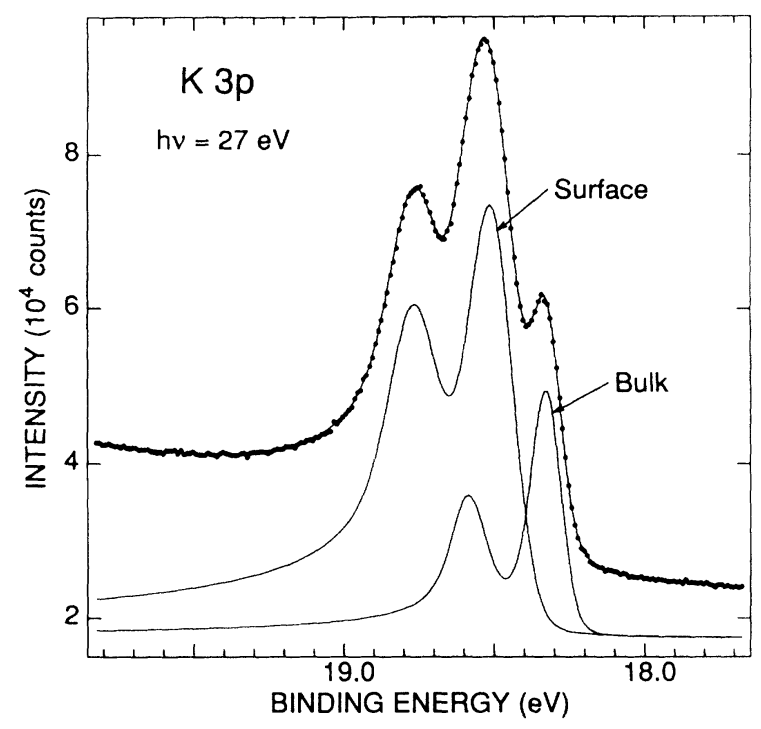

FIG. 1. Analysis of the photoemission spectrum of the $3 p$ region of $\mathrm{K}$ metal. The data, taken with the sample at $172 \mathrm{~K}$, are one of a set used to determine the temperature dependence of the phonon width. The bulk and surface spin-orbit doublets, together with a power-law background (not shown), produce the line through the data points.

contributions from the instrumental resolution $G_{\text {res }}$, is approximately given by

$$
\begin{array}{r}
G_{\mathrm{tot}}^{2}=G_{\mathrm{res}}^{2}+G^{2}(0) \frac{8}{3} \frac{T}{\Theta_{D}}\left[1+\frac{1}{2}\left[\frac{3}{8} \frac{\Theta_{D}}{T}\right]^{2}+\cdots\right] \\
\left(T>\Theta_{D}\right),
\end{array}
$$

where $G(0)$ is the Gaussian width from the phonon broadening at $T=0 \mathrm{~K}$. For $T>\Theta_{D}$, the second term in

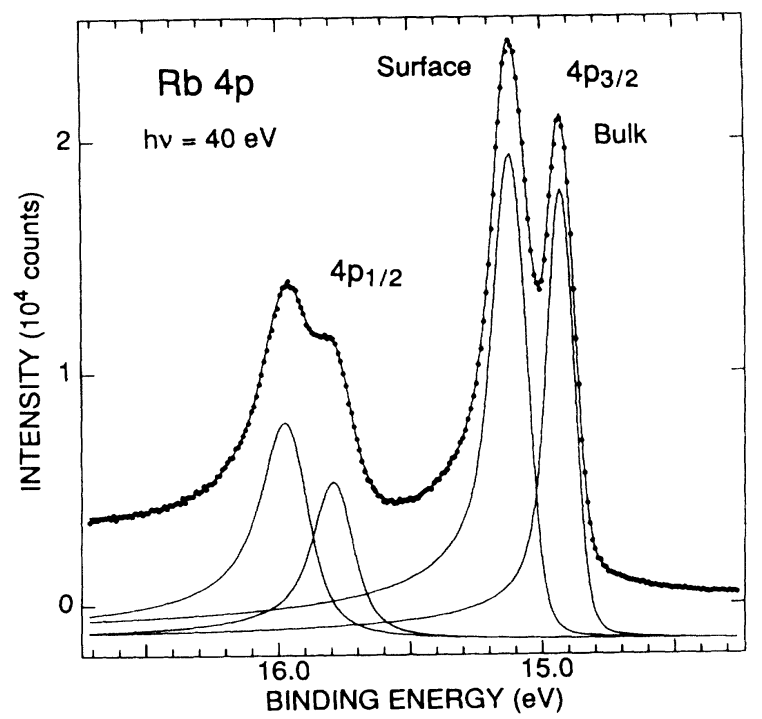

FIG. 2. Analysis of the photoemission spectrum of the $4 p$ region of $\mathrm{Rb}$ metal. The data, taken with the sample at $147 \mathrm{~K}$, are one of a set used to determine the temperature dependence of the phonon width. The components of the bulk and surface spin-orbit doublets are shown separately. 
square brackets is smaller than 0.07 . Such a plot should therefore be linear for temperatures greater than the corresponding Debye temperatures, which for $\mathrm{Na}, \mathrm{K}$, and $R b$ are 155,100 , and $50 \mathrm{~K}$, respectively. The linearized form of Eq. (1), i.e., neglecting the second term in the square brackets, extrapolates to an intercept at $T=0$ equal to the square of the instrumental resolution.

For all three metals, the bulk Gaussian width exhibits the expected linear increase of $G_{\text {tot }}^{2}$ with temperature. This affirms our methodology for obtaining Gaussian widths from the data. The lines drawn through the bulk widths are the results of linear least-squares fits to the data points. The fact that the intercepts of these lines at

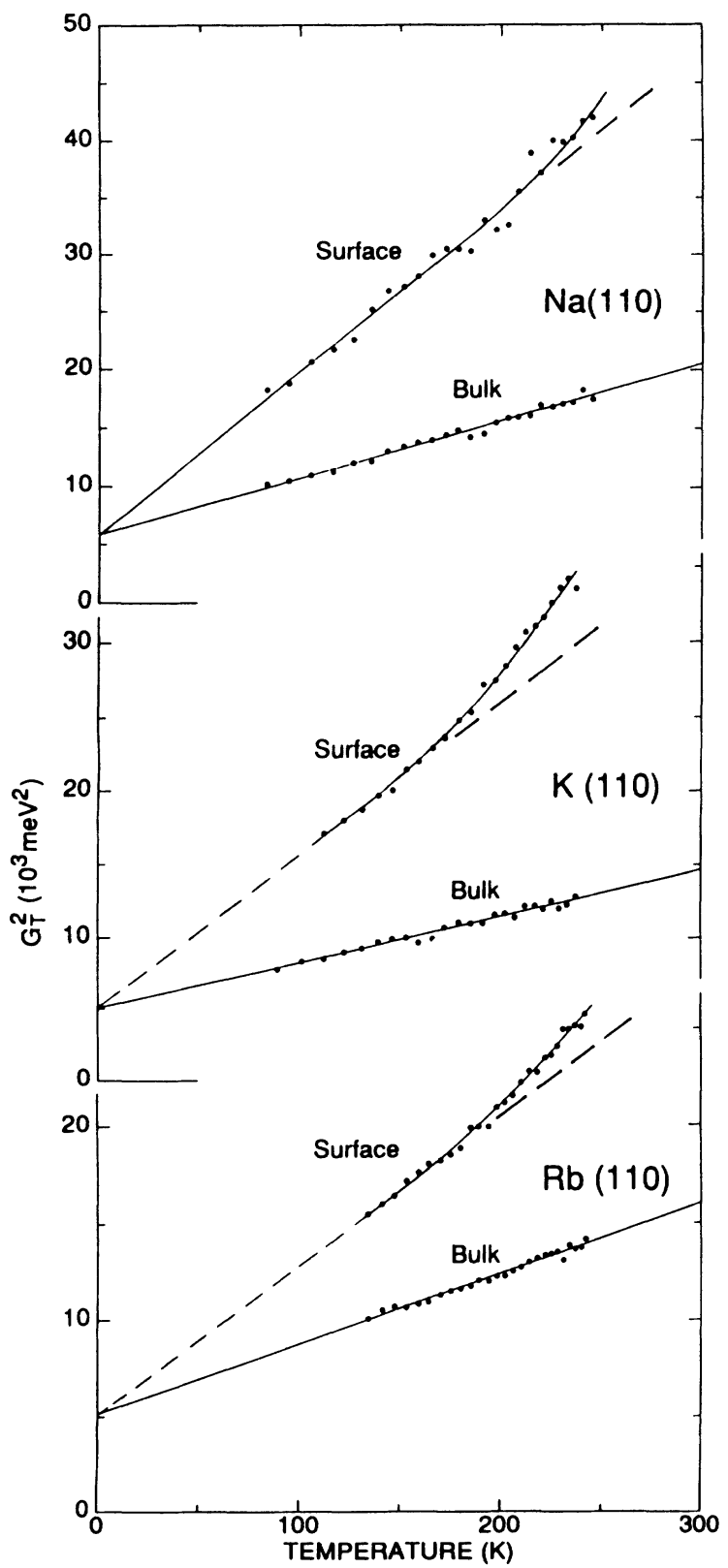

FIG. 3. Square of the total Gaussian width of bulk and surface $n p$ spin-orbit doublets of $\mathrm{Na}, \mathrm{K}$, and $\mathrm{Rb}(n=2,3$, and 4, respectively) plotted against temperature. The straight lines through the bulk data are the results of least-squares fits. The curved lines through the surface data are guides to the eye.
$T=0$ are all close to the square of the instrumental resolution, independently measured from the width of the Fermi cutoff, confirms the validity of Eq. (1).

Because the data for $\mathrm{Na}$ start below $\Theta_{D}$, it is desirable to compare a fit to the data using the more general expression for the phonon width, ${ }^{23}$

$$
G^{2}(T)=G^{2}(0)\left[1+8\left[\frac{T}{\Theta_{D}}\right]^{4} \int_{0}^{\Theta_{D} / T} \frac{x^{3} d x}{e^{x}-1}\right] .
$$

The result is shown in Fig. 4 together with the hightemperature, linearized asymptotic form from Eq. (1). It is clear that a fit with a straight line results in only a small overestimate of the intercept. For K, with a smaller $\Theta_{D}$, the separation between the fit using Eq. (2) and that using Eq. (1) is smaller than the scatter of the data.

The fit to the bulk $\mathrm{Na}$ data in Fig. 4 yields a more reliable value for the zero-temperature phonon width than can be obtained with the high-temperature expansion in Eq. (1). The resulting value of $54 \mathrm{meV}$ is in reasonable agreement with the theoretical estimate of $65 \mathrm{meV} .^{24}$ The extrapolated phonon width at $300 \mathrm{~K}$ from Fig. 4 is $124 \mathrm{meV}$, slightly larger than the semiempirical value of $106 \mathrm{meV}$ obtained by Flynn. ${ }^{24}$ A similar fit to the $\mathrm{K}$ data (not shown) yields a zero-temperature phonon width of $32 \mathrm{meV}$ and a $300-\mathrm{K}$ width of $91 \mathrm{meV}$. The extrapolated phonon width in $\mathrm{Rb}$ at $300 \mathrm{~K}$ from Fig. 3 is $104 \mathrm{meV}$. The room-temperature widths for $K$ and $R b$ are in good agreement with those obtained from soft-x-ray emission edges. ${ }^{25}$ These results for the bulk phonon widths confirm that our method of data analysis successfully separates the instrumental and lifetime widths from the phonon contribution and gives confidence to our analysis below of the surface phonon width.

At higher temperatures, the surface Gaussian widths deviate from the expected linear behavior for all three metals. In $\mathrm{Na}$ this deviation is relatively small, but in $\mathrm{K}$ and $\mathbf{R b}$ the deviations are much more pronounced. This

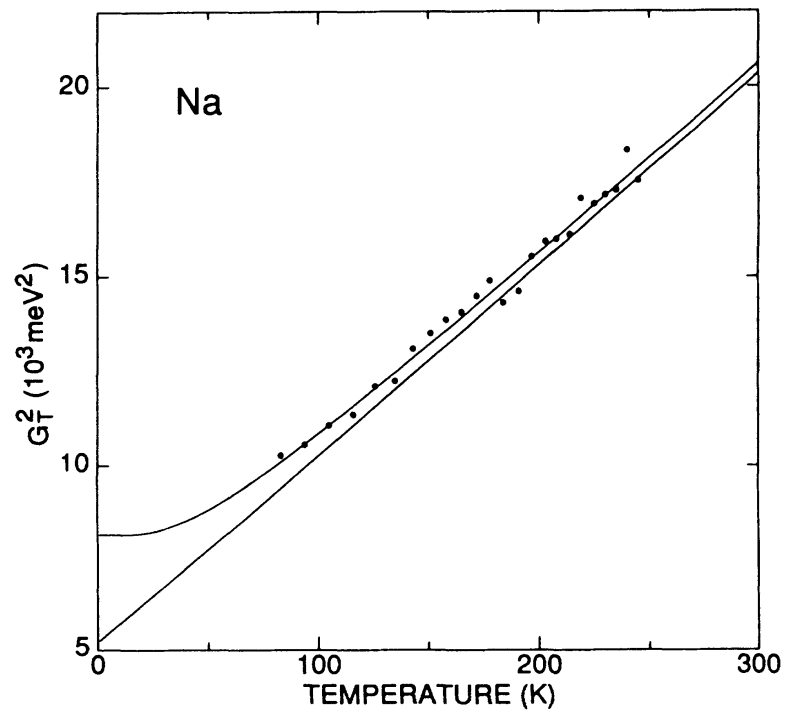

FIG. 4. Fit to the bulk Gaussian width of $\mathrm{Na}$ using Eq. (2) (see text). 
upward curvature is not compatible with Debye theory, in which the square of the phonon width increases linearly with temperature above the Debye temperature. In view of the smaller Debye temperature (larger slope) of the plot of the square of the surface Gaussian width versus temperature, there is no need to invoke Eq. (2), even for $\mathrm{Na}$. In terms of the Debye model, the deviations can be thought of as being due to a decrease of $\Theta_{D}$ for surface atoms at elevated temperatures, and likely involves a softening of the mode normal to the surface. The softening begins around $200 \mathrm{~K}$ in $\mathrm{Na}$ and $150 \mathrm{~K}$ in $\mathrm{K}$ and $\mathrm{Rb}$, or about half the bulk melting point.

The softening at large excitation is a result of the weak restoring force for excursions away from the surface, and so is directly related to the desorption channel of the surface atoms. For $\mathrm{Na}, \mathrm{K}$, and $\mathrm{Rb}$, the vapor pressure reaches $3 \times 10^{11}$ Torr at 300,255 , and $240 \mathrm{~K}$, respectively. Anharmonicity becomes apparent well below these temperatures. The surface anharmonicity is much greater than that in the bulk because the surface potential contains large odd-power terms to express the asymmetry of the restoring force for displacements toward and away from the surface.

\section{CONCLUSIONS}

The phonon widths of core-electron photoemission spectra from atoms in the first atomic layer of alkali metals give evidence for a softening of the vibrational mode normal to the surface for temperatures above one-half the bulk melting point. The softening reflects the asymmetry of the potential well, being shallower for displacements away from the surface than for those toward it. The resulting anharmonic vibrations also correlate with vapor pressure, which in turn depends on the potential-well depth in desorption.

\section{ACKNOWLEDGMENT}

Photoemission research was carried out at the National Synchrotron Light Source (NSLS), Brookhaven National Laboratory, which is supported by the Department of Energy, Division of Materials Sciences and Division of Chemical Sciences.
*Present address: Dept. of Physics, Utah State University, Logan, UT 84322.

${ }^{1}$ A. U. MacRae, Surf. Sci. 2, 522 (1964).

${ }^{2}$ R. M. Goodman, H. H. Farrell, and G. A. Somorjai, J. Chem. Phys. 48, 1046 (1968).

${ }^{3}$ J. M. Morabito, Jr., R. F. Steiger, and G. A. Somorjai, Phys. Rev. 179, 638 (1969).

${ }^{4}$ D. P. Jackson, Surf. Sci. 43, 431 (1974).

${ }^{5}$ S. K. S. Ma, F. W. De Wette, and G. P. Alldredge, Surf. Sci. 78, 598 (1978).

${ }^{6}$ R. S. Williams, P. S. Wehner, J. Stör, and D. A. Shirley, Phys. Rev. Lett. 39, 302 (1977).

${ }^{7}$ C. S. Jayanthi, E. Tosatti, and L. Pietronero, Phys. Rev. B 31, 3456 (1985).

${ }^{8}$ Th. Fauster, R. Schneider, H. Dürr, G. Engelmann, and E. Taglauer, Surf. Sci. 189/190, 610 (1987).

${ }^{9}$ P. Roubin, D. Chandesris, G. Rossi, J. Lecante, M. C. Desjonquères, and G. Tréglia, Phys. Rev. Lett. 56, 1272 (1986).

${ }^{10}$ F. Sette, C.-T. Chen, J. E. Rowe, and P. H. Citrin, Phys. Rev. Lett. 59, 311 (1987).

${ }^{11}$ L. Wenzel, D. Arvanitis, H. Rabus, T. Lederer, K. Baberschke, and G. Comelli, Phys. Rev. Lett. 64, 1765 (1990).

${ }^{12}$ F. Sette, T. Hashizume, F. Comin, A. A. McDowell, and P. H.
Citrin, Phys. Rev. Lett. 61, 1384 (1988).

${ }^{13}$ S. G. Mochrie, Phys. Rev. Lett. 59, 304 (1987).

${ }^{14}$ P. Zeppenfeld, K. Kern, R. David, and G. Comsa, Phys. Rev. Lett. 62, 63 (1989); G. Armand and P. Zeppenfeld, Phys. Rev. B 40, 5936 (1989).

${ }^{15}$ A. P. Baddorf and E. W. Plummer, J. Electron Spectrosc. Relat. Phenom. 54/55, 541 (1990).

${ }^{16}$ D. M. Riffe, G. K. Wertheim, and P. H. Citrin, Phys. Rev. Lett. 67, 116 (1991).

${ }^{17}$ B. S. Itchkawitz, I.-W. Lyo, and E. W. Plummer, Phys. Rev. B 41, 8075 (1990).

${ }^{18}$ S. Doniach and M. Šunjić, J. Phys. C 3, 285 (1970).

${ }^{19}$ G. K. Wertheim, D. M. Riffe, and P. H. Citrin, Phys. Rev. B 45, 8703 (1992).

${ }^{20}$ D. M. Riffe, G. K. Wertheim, D. N. E. Buchanan, and P. H. Citrin, Phys. Rev. B 45, 6216 (1992).

${ }^{21}$ M. W. D. Mansfield, Proc. R. Soc. London Ser. A 346, 539 (1975).

${ }^{22}$ G. K. Wertheim, D. N. E. Buchanan, and J. E. Rowe, Solid State Commun. 7, 903 (1991).

${ }^{23}$ L. Hedin and A. Rosengren, J. Phys. F 7, 1339 (1977).

${ }^{24}$ C. P. Flynn, Phys. Rev. Lett. 37, 1445 (1976).

${ }^{25}$ R. L. Fink, P. N. First, and C. P. Flynn, Phys. Rev. B 38, 5839 (1988). 